Cómo citar este artículo: Campion, M. (2015, Julio- Diciembre). Entre la memoria histórica y el atentado: las relaciones transfronterizas de los indígenas Pastos del Nudo de Waka. Rev. Cient. Gen. José María Córdova 13(16), 243-262

\title{
Entre la memoria histórica y el atentado: las relaciones transfronterizas de los indígenas Pastos del Nudo de Waka"
}

Recibido: 28 de mayo de 2015 - Aceptado: 10 de julio de 2015

Remembering the Past and Remembering the Attacks: Cross-Border Relationship of the Pastos indigenous community in the Huaca Knotch

Sans oublier le passé et se souvenant de l'attentat: les relations transfrontalières des groupes ethniques dans le Noud de los Pastos

Entre a memória histórica e o atentado: as relaçóes transfronteiriças dos povos indígenas no Nudo dos Pastos

Minerva Campion ${ }^{a}$

* Artículo de investigación asociado al proyecto: "La organización indígena transfronteriza en los márgenes del Ecuador. Perspectivas desde la Sierra, Costa y Amazonía”. Universidad del País Vasco, Leioa, España.

a Doctora en Estudios Internacionales; docente en la UniversidadCentral.Comentariosa:MCAMPIONC@ ucentral.edu.co 
Resumen. Se abordan los procesos de integración fronteriza desarrollados por los indígenas Pasto del Nudo de Waka, ubicado entre el espacio fronterizo de Ecuador-Colombia. Para ello, se analiza el significado de la propia frontera para los Pasto y los procesos de fragmentación y de reconstrucción que están ocurriendo en los márgenes de estos Estados. El método utilizado para elaborar esta investigación es el descriptivo-hermenéutico, el cual permite realizar una reflexión acerca de los procesos que ocurren en las fronteras, así como comprender desde la perspectiva indígena las implicaciones que tiene vivir en estos territorios. De este modo, también veremos las formas de integración desarrolladas por los Pasto, su actuar como sujetos políticos y la revalorización de su identidad a través de las actividades transfronterizas.

Palabras claves: atentado, frontera Ecuador-Colombia, Nudo de Waka ${ }^{1}$, pueblo indígena de los Pastos, relaciones étnicas transfronterizas.

Abstract. This article presents the cross-border integration process of the indigenous group Pasto from the Nudo de Waka, located in the borderlands of Ecuador-Colombia. For doing so, we analyse the meaning of the boundary for the Pasto, and the processes of fragmentation and reconstruction that take place in the margins of these States. The method used to carry out this research has been descriptive and hermeneutic. It allows us to do a reflection on the processes happening in the borderlands, as well as understanding the implications of living in that territory through the indigenous perspective. Moreover, we will also take into account the integration dynamics developed by the Pasto, their role as political subjects and the revalorization of their identity through cross-border activities.

Keywords: attack, Ecuador-Colombia border, indigenous Pasto people, Knot Waka.

Résumé. L'article examine les processus d'intégration frontalière développées par des groupes ethniques dans le Nœud de los Pastos, situés à la frontière entre l'Équateur et la Colombie. Dans ce contexte, on analyse le sens de la frontière même pour le groupe ethniques des Pastos, et aussi les processus de fragmentation et de la reconstruction qui ont lieu autour des frontières de ces Etats. La méthode utilisée pour développer cette recherche est de caractère descriptif-herméneutique, qui permet de examiner attentivement les processus qui se produisent aux frontières, ainsi que de comprendre en perspective autochtone les implications de la vie dans ces territoires. Ainsi, nous verrons également les formes d'intégration développées par le groupe ethnique des Pastos, leur capacité d'agir comme sujets politiques et la mise en valeur de son identité à travers des activités transfrontalières.

Mots-clés: attentat, Frontière Equateur-Colombie, groupe ethniques des Pastos, Nœud de los Pastos.

1 N. del E. Nudo de WaKa, o Nudo de Huaca, más conocido como el Nudo de los Pastos, hábitat del grupo indígena transfronterizo de los Pastos que se identifican como un pueblo o etnia indígena que ocupó y ocupa la franja transversal del sur de Colombia y el norte del Ecuador; viven en el conjunto orográfico de montañas escarpadas que amuralla la provincia ecuatoriana de Carchi y lo que es hoy el Departamento de Nariño, a tres mil metros sobre el nivel del mar, cerca donde la cordillera de los Andes que se bifurca en dos ramas al entrar a Colombia: la Cordillera Occidental y la Cordillera Central. La palabra quechua wak'a 'huaca', utilizada por la autora, significaba 'territorio sagrado' en los tiempos coloniales de los Incas del antiguo Perú, y en la actualidad sugiere la vinculación de los Pastos con el territorio ancestral de los pueblos quechuas de los Andes; wak'a, en efecto, significaba en la Colonia tanto lugar sagrado, montaña, templo, tumba, o piedra, como al dios que representaba, como la huaca del sol, o el templo del Sol, y la huaca de la luna, o el templo de la Luna, y otras sacralidades incaicas como animales, momias, o ídolos. Según el lingüista Hugo R. Albor, el quechuismo moderno huaca, o guaca, de / wak'al tiene tres significados básicos: 1) 'dios familiar, penates'; 2) entierro, tesoro, escondrijo; 3) 'alcancía'. Cf. Albor (1972): Apuntes lexicográficos del español hablado en Nariño (Instituto Caro y Cuervo), Thesaurus 27(2), p. 339, s. v. guaca. En Colombia, Bolivia y el Perú guaca se refiere al sepulcro de los antiguos indios, en que se encuentran a menudo objetos de valor, dice el Diccionario de la Real Academia Española. En relación con el sentido antiguo de la palabra wak'a, véase el dibujo 109, sobre abluciones y agüeros del códice peruano ilustrado Nueva Corónica y Buen Gobierno, escrito entre 1583 y 1615 por Felipe Guaman Poma de Ayala [Waman Puma] (ca.1526-ca.1620), dibujante y autor), edición facsimilar de Paul Rivet (París, 1936, Institut d’Ethnologie). Allí el Inca Tupa Yupanqui conversa con los huacas (objetos sagrados) de las distintas regiones al pie de la huaca (templo) Huanacaure (localizado en el cerro del mismo nombre a $12 \mathrm{~km}$ de la actual ciudad del Cuzco). «iWakas sagradas! ¿Quién de ustedes ha dicho: 'No llueva, que no hiele, que no granice’? ¡Hablen! Esto es todo». «No fuimos nosotros, Inca» El mundo de los huacas queda representado como un mundo de alianzas en el que todos se relacionan entre sí. El Inca creía que por medio de las huacas sagradas se podía conocer los hechos pasados y futuros, incluso la llegada de los españoles a América. 
Resumo. Serão abordados os processos de integração transfronteiriça desenvolvidos pelos povos indígenas Pastos no Nudo dos Pastos, localizado entre a área da fronteira do Equador-Colômbia. Para isto, analisa-se o significado da própria fronteira para os Pastos e os processos de fragmentação e reconstrução que têm ocorrido nas fronteiras desses Estados. O método utilizado para desenvolver esta pesquisa é de ordem descritiva-hermenêutica, o qual permite realizar uma reflexão sobre os processos que ocorrem nas fronteiras, bem como de compreender melhor, a partir de uma perspectiva Indígena, as implicações de viver nesses territórios. Assim, veremos também as formas de integração desenvolvidos pelos povos Pastoa, seu agir como sujeitos políticos e a reavaliação de sua identidade através de actividades transfronteiriças.

Palavras-chave: atentado, fronteira Equador-Colômbia, Nudo dos Pastos, povos indígena Pasto, relaçóes transfronteiriças dos povos indígenas.

\section{Introducción}

A lo largo de la Sierra del Ecuador se asientan diversos pueblos indígenas que se agrupan actualmente en la organización ECUARUNARI (Ecuador Runakunapak Rikcharimuy); ${ }^{2}$ de todos ellos solo uno es transfronterizo, el pueblo Pasto, que está ubicado en la frontera norte de Ecuador, en el Nudo de Waka. Desde el pensamiento Pasto, como veremos a continuación, se reivindica la interrelación de la Sierra, Costa y Amazonia, de sus tierras altas y bajas, frías y cálidas y se han elaborado diversos proyectos transfronterizos con el apoyo de actores estratégicos.

Los Pastos, tomando como base la vinculación territorial e identitaria, se encuentran construyendo alternativas de integración fronteriza diferentes a aquellas que manejan los Estados y las instancias supranacionales. Como señala Navarro Wolff (2009) "esta es una lectura de hermandad profunda, es la otra versión y mirada de la frontera” (p. 179); de igual forma, como indica Castro (2011) "no solo es un lugar (topos) o un espacio, sino que es un hábitat donde las interrelaciones entre quienes lo habitan y que por su vivir lo transforman, marcan el ritmo de la vida en el territorio" (p. 35).

De este modo, en la frontera norte se pueden observar dos procesos, uno étnico y otro comercial (Navarro, 2009). En el caso de la vinculación étnica es el pueblo Pasto binacional el que ha comenzado un proceso de recuperación identitaria a través del Plan de Vida Binacional Pasto, liderado por la organización Shaquiñan (Asociación de Cabildo o Autoridades Tradiciones del Nudo de los Pastos), que se estructura bajo diversos ejes: el territorio ancestral y ambiente natural; la cultura ancestral y la educación propia; la soberanía alimentaria, la medicina natural y el desarrollo económico; y el gobierno, administración y justicia.

Cabe destacar también que la presente investigación forma parte de la tesis doctoral realizada por la presente autora en la Universidad del País Vasco sobre los procesos de integración, fragmentación y resistencia que existen en los márgenes del Ecuador. Es decir, hemos investigado las estrategias transfronterizas desarrolladas por los pueblos indígenas de la Sierra, Costa y Amazonía frente a diversos procesos de dominación, en la frontera colombo-ecuatoriana y ecuatoriano-peruana (Campion, 2014).

2 Los pueblos son: Chibuleo, Kañari, Karanki, Kayambi, Kisapincha, Kitu Kara, Natabuela, Otavalo, Panzaleo, Puruwá, Salasaka, Saraguro, Tomabela, Waranka, Pasto y Palta. 
El objetivo del presente artículo es mostrar a los pueblos indígenas, a través del caso del pueblo Pasto, como sujetos activos y sujetos políticos de la vida en la frontera. En este sentido, este artículo es un esfuerzo por transcender el ejercicio antropológico y situar a estos pueblos en la vida internacional.

Por último, cabe recordar que el estudio de las relaciones transfronterizas indígenas entre Colombia y Ecuador no es un tema que se haya abordado en profundidad hasta la fecha. Por ello, un segundo objetivo busca aportar mayor información en este espacio vacío del conocimiento, mostrando las formas de integración, fragmentación y reconstrucción a las que se enfrentan los indígenas Pasto en el Nudo de Waka.

\section{Fragmentaciones, fronteras y territorios en el Nudo de Waka}

Los primeros documentos sobre los espacios ocupados por los pueblos indígenas de la Sierra norte del Ecuador se encuentran recopilados por el cronista Cieza de León. El cronista menciona las provincias de "los Abades", "los Pastos", "los Quillacingas" y "la de Cibundoy" (Groot de Mahecha y Hooykaas, 1991). Estudios recientes han tratado de delimitar estos territorios ancestrales (Calero, 1991; Groot de Mahecha y Hooykaas, 1991; Delgado, 2004). Aunque todos comparten que los Pasto se ubicaban entre los ríos Guaitara y Chota, no hay consenso sobre su extensión hacia los flancos orientales y occidentales (Mamián, 2000). Sin embargo, en términos generales, podemos considerar que el territorio Pasto abarca los siguientes linderos:

[...] por el sur, desde lo que hoy se conoce como el Valle del Chota (Ecuador); por el norte, el lindero natural es el río Guáitara, por el lado sur del sitio conocido como El Pedregal; por el oriente, el lindero son las partes medias (cordillera) del actual municipio de Funes, donde los pasto tenían tarabitas de vigilancia; continúa por la parte alta de Puerres, Córdoba, Potosí, Ipiales; por el occidente el territorio pasto limita con lo que actualmente es el resguardo Ojal la Turbia (desembocadura del río Mayasquer al río Mira), ubicado en el actual municipio de Tumaco; continúa hasta el sitio las Tulpas en el cerro Madroño, lugar de diálogos entre pasto y awá (actual municipio de Ricaurte); sigue por el actual municipio de Mallama y la parte alta del municipio de Santa Cruz; continúa por los municipios de Providencia, Guaitarilla e Iles, hasta volver a encontrar el río Guáitara. (Guerrero, 2008, pp. 181-183)

Aunque una forma de comprender el territorio de los Pasto proviene de la perspectiva anterior, también se puede definir a partir de la concepción territorial de los Pasto que se encuentra centrada en el Nudo de Waka o el Nudo de los Pasto. Desde esta concepción del territorio logran trascender la frontera de los Estados, es decir, recurren al ambiente - a la Madre Tierra- y a la identidad indígena-Pasto- que los alberga al interior de ese Gran Macizo.

Para todos es conocido que para el pueblo de los Pasto, habitantes del nudo de los Pastos, el territorio no ha tenido fronteras ni límites. Hasta ahora, recorremos los páramos, los ríos, y para nosotros las fronteras impuestas por el general Flórez y el general Mosquera por medio de la guerra nunca nos han obstaculizado. Sin embargo, hemos sufrido la degradación de lado y lado. (Tarapues, 2009, p. 81) 
Como consecuencia de la fragmentación de su territorio entre dos Estados y por ende la aplicación de diferentes formas de organización, en Colombia se agrupan en resguardos y en Ecuador en comunas. La organización comunal en Ecuador comenzó en 1937 con la Ley de Comunas, donde los pequeños pueblos de indios pasaron a llamarse comunidades y los cabildos pasaron a denominarse comunidades campesinas (Lucio y Castro, 2011). En Colombia la Constitución de 1991 fue fundamental para el reconocimiento de las demandas indígenas que los Pasto reivindicaban desde 1970, principalmente a través del Movimiento de las Autoridades Indígenas del Sur Occidente (Tarapues, 2013). Aun así, hay que recordar que la defensa del territorio entre los Pasto proviene de la larga duración como se ha documentado en informes del periodo colonial y republicano (Guerrero, 2008, p. 185).

Sobre los años de 1820 y 1850 se fijan los límites internacionales a la fuerza, por los liberales y los conservadores, y mediante la guera colocaron las fronteras y es así que el territorio ancestral y cultural queda descuartizado quedando los Andinos Pastos en un lado y en el otro de las repúblicas. (Navarro, 2009, p.173)

La fragmentación territorial con referencia a los Pasto se sitúa más allá del establecimiento de una línea divisoria que ha llegado a incidir en la pérdida del territorio y de la identidad. Hasta la fecha la identidad Pasto de Colombia se encuentra mejor valorada que la ecuatoriana (Romero, 2010). Además, los Pasto de Colombia consideran que pertenecen a la "Nación Pasto", concepto con una connotación más arraigada al territorio, puesto que "es una categoría del estado que implica sentido de pertenencia a un territorio soberano" (Lucio y Castro, 2011). Los Pasto de Ecuador casi han desaparecido (Tarapues, 2006), se auto-reconocen como "Pueblo" (al interior de la Nacionalidad Kichwa), en vez de considerarse "Nación” y se asocian al campesinado (Tarapues, 2013, 2009).

En este sentido, y con relación a lo anterior, observamos que en el año 1989 la CONAIE (Confederación de las Nacionalidades Indígenas del Ecuador) destacaba lo transfronterizo de los pueblos y nacionalidades de Ecuador, pero consideraba que la Sierra venía ocupándose principalmente por los Kichwa. En relación a este tema Pineda y Krainer (2012) plantean que "la 'kichwización' del mundo indígena y ancestral ecuatoriano, opera como una hegemonía similar a un 'proyecto de construcción nacional', que invisibiliza a muchos para visibilizar a pocos, y que crea patrones y modelos que operan de un modo homogenizador" (p. 16).

En este aspecto, Estacio (2010) retoma la palabra de los mayores y señala que "así como está dividido nuestro territorio también puede estar dividido nuestro pensamiento, porque eso es el reflejo de lo que la gente piensa, divide también el territorio" (p. 24). Sin embargo, podemos considerar que el auto reconocimiento Pasto en Ecuador, ha venido influenciado por dos procesos, uno a nivel estatal (Romero, 2010) y otro a nivel transfronterizo.

Nosotros, en un camino trazado en procesos de reivindicación de derechos, prácticamente logramos aglutinar a un conjunto de comunas que en una primer instancia nos hemos autodenominado "campesinos", pero siguiendo más adelante en la planificación de acciones y actividades hacia el desarrollo hemos tenido que unirnos a las organizaciones nacionales; y es cuando en el proceso de unirnos a las organizaciones nacionales se toma la lección de la historia en lo que respecta a la identidad: recuerdo, y no he de olvidar nunca, en una reunión donde pedíamos la admisión de nuestra organización a ECUARRUNARI; allí pues en las hojas de inscripción nos habían tomado la lección 


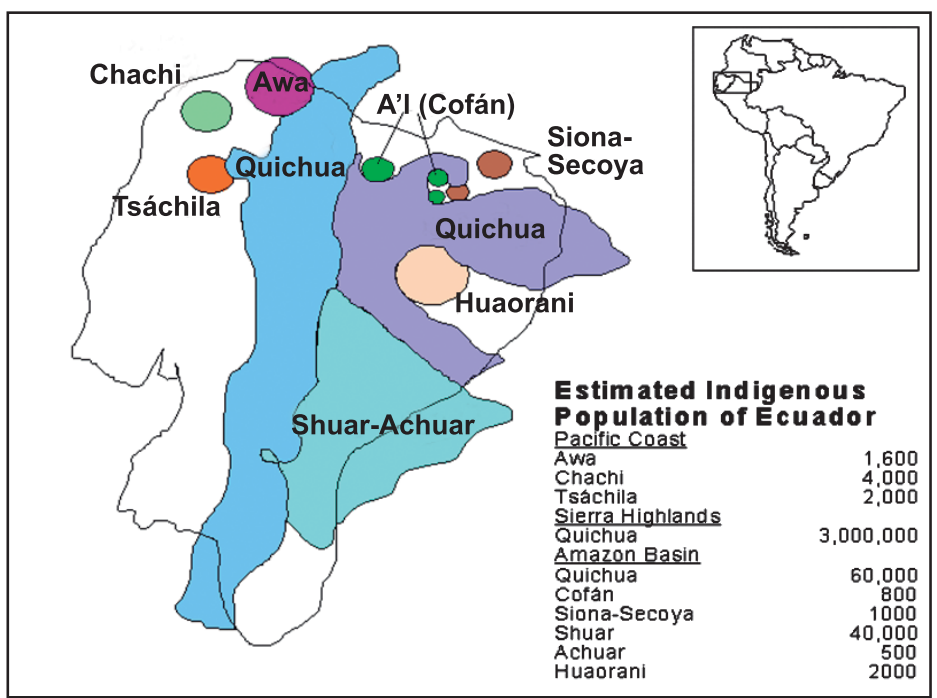

Figura 1. Mapa de nacionalidades (trans)fronterizas del Ecuador según CONAIE Fuente: CONAIE (1989, p. 284).

y nos decían de qué nacionalidad somos y a cuál pueblo pertenecemos; el grupo de etnia Pasto de Ecuador que acudimos a ese evento nos miramos todos a los ojos y por unanimidad dijimos 'nosotros somos Pastos'. (Romero, 2010, p. 74)

La revaloración identitaria de los Pasto en Ecuador también se puede vincular al proceso binacional y de integración fronteriza que están desarrollando. En este contexto se entiende por ejemplo que en 2011 se realizase el primer auto reconocimiento identitario de los indígenas Pasto de la comuna La Libertad (cantón Espejo, Carchi) con la participación de los Pasto del Nudo de Waka (La Hora, 2011).

En el lado ecuatoriano la cultura Pasto ha sido golpeada, casi desaparecida y sus descendientes se han planteado el propósito de difuminar la frontera, para concebir y mirar al gran macizo como eje de una planeación binacional. [...] En Colombia, en cambio, la identidad Pasto ha sobrevivido, actualmente hay cerca de 91 mil indígenas en 21 resguardos indígenas. (Tarapues, 2006).

Estacio (2010) hace referencia a la importancia de reivindicar la memoria histórica pero también el atentado (es decir, la fragmentación) que ha afectado a la cultura y a la identidad, por lo cual tienen que rescatarse los "encuentros frente al agua, frente a las montańas, los ritos, la sacralidad" (p. 24). A pesar de las fragmentaciones causadas por las empresas extractivas, los actores armados, la construcción de carreteras y la propia frontera, aún se mantiene y se está reconstruyendo su cosmovisión y sus prácticas culturares, productivas, de salud y educativas.

En este sentido, el fragmentalismo ha surgido como uno de los principales conflictos para los pueblos originarios. El fragmentalismo debe ser entendido como la ambición del hombre por la explotación del territorio y por la obtención de recursos, ello aunado a la imposición de linderos y de las 
reservas y resguardos indígenas por parte del Estado colombiano, lo cual ha generado el quebrantamiento del principio de comunitarismo entre estos pueblos así como la cultura de los mismos. (Tarapues y Rico 2012, p. 69)

Todas estas cuestiones de reconstrucción del territorio como unidad holística, albergados bajo la concepción del Gran Macizo del Nudo de Waka, se están debatiendo en los encuentros, talleres y mingas de pensamiento a nivel transfronterizo, mediante los cuales se construyen "historias otras" que emergen de una territorialidad diferente y de unas fronteras diferentes, al mismo tiempo que fortalecen la identidad a ambos lados del límite artificial.

Lo que buscamos con estos encuentros, con estos talleres, básicamente es abordar la temática que tiene que ver con pueblos indígenas. [...] Revisando la historia, básicamente nuestros pueblos han sido divididos con fronteras impuestas por gobiernos tal vez ajenos a nuestras realidades; por ejemplo el pueblo de los Pasto históricamente ha tenido un solo territorio en el conocido nudo de la Guaca, pero a partir de los tiempos de la república y la colonia este ha sido dividido; así nuestro pueblo, nuestra cultura, nuestro territorio, nuestro ambiente, nos dicen, están entre Colombia y Ecuador. [...] Por ello se ha querido abordar este tema en estos encuentros. Decíamos que es importante construir una palabra de unidad que amase en ese sentido, o que busque entretejer o tejer la realidad de los pueblos no divididos, pueblos que tienen una sola cultura, una sola identidad, un solo proceso organizativo. Todo esto puede aportar a lado y lado, en este caso, concebida en los tiempos actuales por las naciones, en el marco de tener una naturaleza, un territorio que aporte al buen vivir o al nuevo amanecer que nosotros hemos venido hablando. (Estacio, 2010, p. 20)

Sobre la cantidad de habitantes Pasto del Nudo de Waka no hay cifras claras. Aunque el censo del DANE del 2005 de Colombia señala que existen 129801 (Ministerio de Cultura, 2010), Lucio y Castro (2011) argumentan que en el caso colombiano el censo está manipulado por intereses políticos y en el caso del Ecuador aún no hay censo sobre los Pasto.

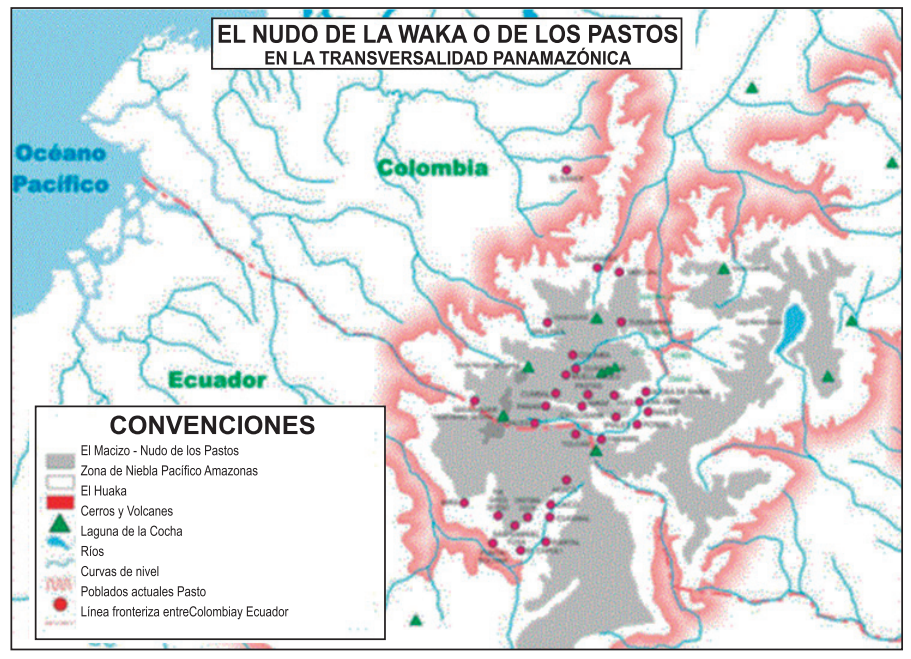

Figura 2. Mapa de El Nudo de Waka o de los Pastos Fuente: Tarapues, et al. (2012). 


\section{Entre la memoria histórica y el atentado: frontera, intercambio y procesos de integración}

En las fronteras andinas de Ecuador-Colombia se ha desarrollado una importante red de carreteras por donde transcurre el comercio (Rivera Flórez, 2010), donde existe una gran presencia de las instituciones estatales y donde la sociedad civil tiene relaciones transfronterizas consolidadas (Carrión, 2010). Tulcán, capital de Carchi, tiene lazos estrechos con Ipiales, municipio colombiano de frontera y ambas tienen una vinculación cultural transfronteriza antigua (Carrión, 2011; Velasco, 2009). Se encuentran conectadas por el Puente Internacional de Rumichaca, donde se mueve "el comercio, la comunicación y la vinculación humana" (Carrion, 2011, pp. 57-58). Recientemente, en el marco de la IIRSA (Iniciativa para la Integración de la Infraestructura Regional Suramericana), se están realizando obras para la construcción de un nuevo Puente Internacional así como la instalación de un CEBAF (Centro de Atención Binacional Fronterizo), pero esto lo analizaremos con mayor profundidad a continuación.

También existen iniciativas transfronterizas relacionadas con la cultura que se han realizado a través de organismos públicos y privados de ambos lados. En este caso son de destacar el "Frente Binacional de Gestión Cultural Pasto" y el proyecto "Legado del Pueblo Pasto como catalizador de una identidad binacional con proyección al mundo", promovido por CODESTAAE de Ecuador y la Fundación y Museo Juan Lorenzo Lucero en Colombia. En el marco del Plan Adelante Narińo (2008-2011) se encontraba también la iniciativa "Plan Frontera sur para el Desarrollo, la Paz y la Integración" que promovía la "formulación e implementación de planes binacionales para comunidades indígenas y afro" así como "encuentros de culturas andinas y del pacífico" (Obando Eraso 2009, p. 70). Asimismo, se ha propuesto en Ipiales la creación de la Comunidad Binacional Transfronteriza con el fin de impulsar el comercio de un lado al otro de la frontera (Arturo, 2013).

A pesar de que en las instituciones municipales se ha comenzado a utilizar "lo indígena" como un factor de integración en la frontera, Tarapues (2013) señala que se les considera sobre todo sujetos folclóricos, es decir, que "se ignora la nación Pasto sujeto histórico, político y cultural, sustancial, binacional del territorio y ambiente Waka; solo se lo reconoce en el discurso y como patrimonio cultural" (Lucio y Castro, 2011, p. 103). Además, desde el establecimiento colonial en territorio Pasto se vieron alteradas sus formas de vida tradicionales que se expresan en la actualidad a través de las formas de organización del territorio basado en el Estado-nación y a través de la tenencia de la tierra.

El pensamiento sistémico que guiaba a la cultura de nuestros mayores, el cual involucraba los organismos, los sistemas sociales y la Madre Naturaleza, actualmente se ignora y se impone la fragmentación, la imposición de lógicas institucionales de ordenamiento territorial, las divisiones político-administrativas (municipios, departamentos y nación), los parques naturales, entre otros. Es así que los territorios ancestrales quedaron divididos por límites que son divisiones ficticias o artificiales para los pueblos originarios. Los síntomas del fragmentalismo son visibles en la tenencia de la tierra, unos pocos con el latifundio y las comunidades originarias con el minifundio, siendo este una creciente problemática que se dirige al colapso. El minifundio es una expresión del individualismo y la cultura colonial, porque antes del ingreso de la colonia a nuestros territorios, los 
andinos no poseíamos la Madre tierra en el sistema individual. Es triste ver que en el minifundio hoy se expresa la extrema pobreza; pobreza económica, pobreza cultural, pobreza mental y pobreza espiritual. (Ministerio de Ambiente, 2012, p. 81)

En contraposición al estudio de Andrade (2002), que concluía que no pudo comprobar la existencia de relaciones culturales y sociales entre Carchi y Colombia, Ramos (2008) demuestra (mediante un exhaustivo trabajo de campo en Carchi) que los vínculos culturales y sociales son fuertes y que se llevan a cabo diversos eventos deportivos, sociales y religiosos con los colombianos, en las ferias o mercados. Además, aunque el paso fronterizo principal de la Sierra sea el puente internacional Rumichaca, los caminos vecinales y los pasos fronterizos — legales o ilegales, que comunican las parroquias de la Sierra entre Ecuador y Colombia — han sido construidos a partir de las Mingas o los trabajos voluntarios comunitarios. En este sentido, se muestra el interés de las poblaciones locales por vincularse de manera transfronteriza (Vieira, 2008, p. 435).

Una segunda crítica que se le puede hacer a Andrade (2002) es que según el autor Carchi es "étnicamente homogénea y sin presencia significativa de movimientos etno-políticos". Aunque es cierto que la provincia está compuesta principalmente por habitantes mestizos, de acuerdo con lo que veremos a continuación sobre el Pueblo Pasto, los indígenas son sujetos etno-políticos con propuestas de integración fronteriza propia que han trabajado a partir de encuentros binacionales y mingas de pensamiento, incluso cuando los Estados habían roto sus relaciones diplomáticas.

Los Estados han dado históricamente más importancia a la integración económica (Hocquenghem y Durt, 2006) que a la social y, además, los proyectos con orientación hacia lo cultural y ambiental no están articulados ni existen políticas públicas que estén dirigidas a las "necesidades y demandas de territorios comunitarios binacionales" (Lucio y Castro, 2010, p. 35). Con referencia a la integración en la frontera norte, Navarro Wolf (2009) señala dos tipos posibles de hermandad transfronteriza: la primera cultural, ancestral, cosmogónica y consanguínea (como la de los Pasto); y la segunda, comercial y de parentesco que proviene del periodo colonial y republicano.

[...] la hermandad cosmogónica y consanguínea es más estrecha que la hermandad comercial, esta última es de los Estados mediante los tratados, como la Comunidad Andina de Naciones -CAN-, etc. En este último caso solo es una hermandad en relación de las empresas y el mercado, más no el verdadero sentir de los pueblos, como culturales y ancestrales y como descendencias coloniales. (Navarro, 2009, p. 178)

Con referencia a esta hermandad cosmogónica y consanguínea los Pasto destacan la importancia del Convenio 169 de la OIT (Organización Internacional del Trabajo), en particular los artículos sobre los pueblos indígenas transfronterizos para que "se puedan generar iniciativas, en este caso de lado y lado del río" (Estacio, 2010, p. 27).

La carencia de una visión integral y del territorio Waka y la persistencia, subyacente, de un nacionalismo a ultranza que enfatiza en la división fronteriza, impide desarrollar un concepto coherente de integración binacional, la cual se sustantiva como integración comercial. La integralidad generalmente se concibe como la superposición de aspectos como la salud, la educación, el empleo, el ambiente, entre otros aspectos. Desde esta perspectiva, la situación económica y social de los muni- 
cipios del Nudo está determinada en diferentes niveles por su condición de zona de frontera nacional. La frontera aparece entonces más que una posibilidad de encuentro de tradiciones culturales, históricas y naturales compartidas, como una línea que afecta negativamente a las comunidades que habitan en torno a ella. (Mamian e Inuca, 2011, p. 7)

A pesar de la mayor institucionalidad y presencia del Estado en la Sierra, los Pasto manifiestan que esta región sigue siendo periférica y de escaso interés para el Estado (Tarapues, 2013). Es por este motivo que las iniciativas transfronterizas han tenido que ser promovidas en las comunidades y desde el proceso binacional Pasto.

[...] y tanto más que en vano nuestras constituciones nacionales decían que se debe promover el desarrollo de la zona de frontera; pero eso se quedó en meros pronunciamientos. [...] la única parte que vemos es que los únicos que vamos a poder desarrollar esos objetivos, esos sueńos que constan en nuestras constituciones, somos los propios pueblos. (Romero, 2010, p. 74)

Rivera Flórez (2010) señala que la construcción de una “infraestructura vial adecuada contribuirá a que las dinámicas transfronterizas superen la ilegalidad y se desarrollen alrededor de los intercambios comerciales binacionales". En este aspecto apuntar a dos cuestiones: en primer lugar, es debatible que la ilegalidad se supere a través de la creación de carreteras — que supone también la instalación de gasolineras o aduanas, por ejemplo—; y, en segundo lugar, hay que considerar qué se entiende por ilegal y qué no.

En cuanto a la primera cuestión, las poblaciones de frontera consideran que cuando el Estado o la CAN (Comunidad Andina de Naciones) interviene, además de destruir la integración espontánea, los proyectos desarrollados para impulsar el comercio o los megaproyectos de integración, "anulan las funciones tradicionales de las poblaciones locales, ligadas a las aduanas, al trasbordo y a la protección nacional" (Ramírez, 2008b, p. 358). Además, la construcción de carreteras no tiene porqué ser beneficiosa, en primer lugar porque puede encoger los territorios indígenas, y en segundo, porque implica puestos fronterizos, aduanas y, por lo tanto, más control de pasaportes.

Las comunidades han denunciado que en el territorio indígena se están construyendo grandes estaciones de gasolina sin intervención de la autoridad indígena, violando el derecho al territorio y a la consulta previa. Adicionalmente, la Alcaldía Municipal de Ipiales, en convenio con Invías, tiene proyectada en territorio indígena la construcción de un terminal de carga internacional, lo que a su vez conlleva la expansión del casco urbano de Ipiales sobre territorio de resguardo, (Martínez y Houghton, 2008, p. 245)

En el caso de la construcción de macro proyectos como la IIRSA, Martínez y Houghton (2008) hacen hincapié en que "la estructuración para la exportación altera fundamentalmente las regiones socioculturales creadas por los pueblos indígenas" (p. 232). A través de la "Conexión Venezuela (Caracas)-Colombia (Bogotá)- Ecuador (Quito)" se van a implementar dos proyectos en territorios indígenas: el CEBAF en Tulcán-Ipiales y la vía Popayán-Pasto-Rumichaca. Además, junto con este proceso existen intereses de explotación minera y de instalación de cuarteles en Mallama, Ricaurte, Junín, Guayacana, Llorente, La Victoria, Chiles y Cumbal (Martínez y Houghton, 2008, p. 245). 
Viene de otro elemento y es la mirada del Estado orientado hacia la riqueza del territorio; en el caso del territorio de los Pastos, hay hitos definitivos para los próximos años: agua, energías (Zona de Azufral), minería (Territorio del Gran Mallama), la riqueza ecosistémica y biodiversidad del bosque tropical en la zona limítrofe del Putumayo y Nariño (Territorio del Gran Pueblo Mayor de Males); escenario en disputa que tiene consecuencias: desplazamiento en las zonas fronterizas como del Resguardo Cumbal y el Mayasquer que son los más impactados en este tema: esta también el Reguardo de Sande en el pie de monte y de algunos asentamientos indígenas en las zonas cercanas a Guachavez y el municipio de Samaniego. (Calpa, 2011)

Con referencia a las empresas extractivas los Pasto se reunieron en el "Primer Foro Minero del Pueblo Indígena de los Pastos” en 2011. En él adelantaban que las alteraciones realizadas en el ambiente desestabilizan el equilibro del territorio, y en consecuencia el de las comunidades "afectando directamente al nivel cultural, ambiental, económico y político organizativo" (Gobernadores del Pueblo Pasto, 2011).

La minería, la extracción de petróleo, la extracción de minerales, gas, energía (geotermia), madera, las represas y las grandes carreteras, generan graves problemas ambientales al territorio sagrado, además de la pérdida de cultura, conocimientos tradicionales y modos de vida, que a menudo tiene como resultado la explotación desmedida e irracional de los elementos de la Madre Tierra, conflictos, desplazamiento forzado, una mayor marginación, aumento de la pobreza, declinación en la salud de sus pobladores, concentración de la riqueza para unos pocos y pobreza en las comunidades originarias, la apropiación de la materia prima para sus fines productivos en fortalecimiento del capitalismo. (Ministerio de Ambiente, 2012, p. 94)

La segunda cuestión que mencionábamos antes, sobre las características de las zonas de frontera, se refiere a la tensión de legalidad e ilegalidad existente en ellas. En este sentido, muchos de los intercambios comerciales son considerados contrabando por el Estado, mientras que la población local los considera prácticas naturales (Ramírez, 2008a; Grimson, 2000). Con relación a ello, Montenegro (2008a) señala que las instituciones estatales de Colombia y Ecuador aplican las mismas regulaciones comerciales a los espacios de frontera que a los productos que provienen de lugares lejanos. Asimismo, Espinosa (2008) critica las definiciones de comercio lícito e ilícito de los espacios fronterizos ya que al parecer cuando se habla sobre comercio hormiga como estrategia de supervivencia se le tiende a catalogar como ilegal.

Parece que lo lícito es cuando se reúnen las cámaras de comercio de un país y llegan a acuerdos con las cámaras de comercio de otro país. ¿Entonces el comercio formal, el comercio mayorista, ese que de alguna manera acata la mirada institucional y de los Estados, es el lícito y el otro es simplemente ilícito? La pregunta que está en la base es que el comercio hormiga, el que se desarrolla en los términos y límites de la sobrevivencia, ese comercio que de todas maneras es una estrategia de supervivencia para las poblaciones de frontera de uno y otro lado, ese comercio ¿por qué tiene que ser considerado como ilícito? ¿Por qué quien comercia con las FARC con el ELN, incluso con los mismos narcotraficantes, en ese comercio hormiga que es la única alternativa para sobrevivir, tiene que ser considerado ilícito?, ¿por qué ese comercio se criminaliza y no otros comercios que sin duda alguna en este contexto también afectan a las cámaras de comercio y al comercio mayorista? (Espinosa, 2008, p. 392) 
De todas maneras, aunque existan grupos irregulares (FARC-EP, ELN) y redes de delincuencia organizada) es la zona de frontera más estable y poblada en comparación a las de la Costa Pacífica y Amazonia (Carrión, 2011). Sobre este tema es relevante la aportación de la investigación realizada por Ramos (2008) desde varias perspectivas. En primer lugar, la población local de Carchi no identifica gente desplazada o refugiada (p. 181); en segundo lugar, los guerrilleros o guerros no son actores nuevos — como suelen ser caracterizados- y se encuentran integrados en las dinámicas de la frontera mucho antes de la implementación del Plan Colombia (p. 184). Asimismo, la población local de las zonas fronterizas de Carchi afirmaba que los guerros han contribuido a resolver altercados entre los habitantes (Espinosa, 2009; Ramos, 2008) y han participado en los acuerdos transfronterizos de comercio local, como por ejemplo el del gas envasado, controlando a su vez que el acuerdo se cumpliera (Ramos, 2008, p. 162).

De hecho, el único actor que se considera de aparición reciente en esta zona de frontera (a partir de 2006) son los paramilitares de las Autodefensas Unidas de Colombia-AUC (Ramos, 2008, p. 184). En este sentido, actualmente "los terratenientes y los gremios como el ganadero se han rodeado de grupos paramilitares, quienes continúan con las amenazas y los hechos violentos contra las comunidades y los líderes" (Guerrero, 2008, pp. 186-187).

\section{Una integración fronteriza alternativa: de los primeros encuentros a la creación del Plan Estratégico Binacional del Pueblo Pasto}

Aunque el proceso transfronterizo Pasto venía gestándose desde 2003 (Romero, 2010, p. 74), el Primer Encuentro Binacional del Pueblo Pasto tuvo lugar el 21 de junio del 2005 (solsticio de verano) unificando el pensamiento Pasto y el Encuentro "en relación con el cosmos y las enseñanzas de la naturaleza” (Estacio, 2010, p. 27). Además de la participación de los cabildos y comunidades indígenas de Ecuador y Colombia, también estuvieron presentes los gobiernos locales y algunas ONG.

Nos encontramos como Pastos en el año 2005 tratando de hacer esos encuentros no simplemente como una agenda de trabajo sino encontrándonos en relación con las cosmovisiones o en relación con los tiempos del sol y la luna, buscando la cosmovisión y fortalecernos en ese sentido. Nos encontramos en el 2005 en el solsticio de verano el 21 de junio y allí se buscaba encontrar palabra, encontrar pensamiento, encontrar un sueño, encontrar, como decían otros, los caminos para el desencanto o para mirar ese otro espejo que no deja ver nuestra realidad. Allí se hicieron algunos acuerdos, se compartió la medicina tradicional y donde también se levantaron algunos bastones de mando para compartirse como pueblos. (Estacio, 2010, p. 26)

Desde esa fecha, el proceso de reunificación Pasto se ha fortalecido y ha tenido el apoyo de actores estratégicos como los municipios, las ONG y otros organismos internacionales, con lo que finalmente han construido desde su perspectiva el Plan de Vida del Pueblo de los Pasto (Lucio y Castro, 2011). De este modo, se han posicionado frente a los Gobiernos de Colombia y Ecuador con propuestas que surgen de "su propia manera de ver las cosas y que están dirigidas a atender aquello que es necesidad de sus comunidades y en toda la frontera” (Montenegro, 2008b, p. 395). 
[...] los documentos producidos por y desde las expresiones de las comunidades Pasto, que tienen como voz y presupuesto el "Plan Estratégico Binacional para el Fortalecimiento Cultural, Natural y Ambiental del Nudo de los Pastos", suponen la propuesta política de refundar la nación Pasto sobre la base del territorio Waka y con una perspectiva binacional que convierta la frontera nacional-estatal en unión. (Mamian e Inuca, 2011, p. 8)

Este proceso de integración busca la construcción del espacio binacional de los Pasto para "recuperar tierras, gestionar los recursos naturales y ejercer una autoridad político administrativa propia" (Tarapues, 2006, p. 134).

La recuperación cultural y territorial del Nudo de Waka ha sido elaborada a través de diversos Encuentros, Talleres o Mingas de Pensamiento, así como a través de las fiestas del Inti Raymi (fiesta al Sol) y Kolla Raymi (fiesta a la Luna), que se celebran en Ecuador y en Colombia, respectivamente, con el objetivo de trascender la división fronteriza. Asimismo, reiteramos lo dicho con anterioridad, por medio de estos Encuentros Binacionales cara a cara se intercambian experiencias que han permitido que los Pasto del Ecuador reconsideren su ethos y comiencen a identificarse con la "Nación Pasto".

[...] hasta que en el 2005 decidimos armar ese primer encuentro en el marco del Inti Raymi. Pero esa fiesta no solo fue fiesta para lo tradicional, fue una fiesta también que estuvo marcada por la minga del pensamiento donde empezamos a hablar de nuestro pasado como histórico, y nos habíamos dado cuenta de que había, lamentablemente, la división política de nuestras repúblicas que nos habían separado por cerca o alrededor de unos quinientos años; y nosotros al volver nos habíamos vuelto a reunir; y en ese proceso de volvernos a reunir estamos pensando muchas cosas que hacer: Primero la reconstrucción de la nación de los Pastos. Alguien decía o aquí se mencionaba la estadística; pues bueno, en el lado de Colombia hay un orgullo de pertenencia a ser indígena, en el lado nuestro [Ecuador] ocurre lo contrario, y son justamente dos procesos de dominación o hablemos de tres procesos de dominación que tuvimos que sufrir: la invasión Inca, la invasión española y las guerras de la independencia, que definitivamente marcaron a nuestra nación en el lado ecuatoriano. (Romero, 2010, p. 74)

Este proceso de reconstrucción se consolidó además a partir de septiembre de 2008, momento en el que los Estados de Colombia y Ecuador se encontraban en medio de la ruptura de relaciones diplomáticas (Ramírez, 2010, p. 36). En este sentido, lo que ocurre con los Pasto del Nudo de Waka difiere de las posiciones políticas del Estado central (Estacio, 2010, p. 24), ya que estos lugares tienen sus fronteras y territorialidades propias (Santacruz, 2009). Efectivamente es desde esta territorialidad propia donde adquieren sentido las propuestas y las iniciativas de las organizaciones Pasto para reconstruir el territorio del Nudo de Waka, es decir, a través de la compresión del territorio como un organismo vivo que no puede ser dividido (Tarapues, et al., 2012).

Observamos el territorio fragmentado desde hace 517 años por la cultura de Occidente. El hombre de la concepción occidental se colocó límites para desde allí cercar su visión de planeación. Por eso, para nosotros el territorio como organismo vivo no solamente debe entenderse en lo binacional sino en lo panamazónico. El territorio de los Andes en el cual estamos asentados, es un territorio articulado con lo pacífico y lo amazónico [...]. Porque en todos los planes de desarrollo que se escuchan nunca se tienen en cuenta los espíritus que para nosotros son muy importantes [...]. El hombre de la concepción occidental no mira esto, no se ha escuchado esto aquí. El fragmentalismo de los límites 
de las repúblicas y de divisiones político-administrativas para nosotros, no ha dejado pensar en un desarrollo de región. Por eso en el plan estratégico binacional del pueblo los Pastos hacemos énfasis en este tema. (Tarapues 2009, p. 82)

La Panamazonía desde la cosmovisión del territorio de los Pasto viene dada por la verticalidad y la transversalidad del ambiente. Transversalmente está formada por las regiones de la Sierra, Costa y Amazonia; y verticalmente, se constituye hacia el interande "por las alturas paramunas, nevadas y volcánicas de las dos cordilleras, los altiplanos y terrazas de las tierras medias y las tibias y calientes hacia el cauce de los ríos Chota y Guáitara" y hacia "los flancos exteriores, por las tierras altas de una y otra cordillera, las pequeńas mesetas medias y las tierras bajas del piedemonte amazónico y pacífico, respectivamente" (Mamian, 2000).

El nudo de los Pastos comprende Ecuador y Colombia y no solamente abarca el pueblo de los Pastos sino a otros pueblos como los Inga, los Kamentza del Sibundoy, los Quillasinga, los Cofan. Ojalá pues en el futuro podamos integrar a todos estos pueblos alrededor de conservar el nudo de los Pastos, el nudo montańoso, ese el propósito que hemos iniciado [...]. (Valenzuela, 2007, p. 210)

Navarro Wolf (2009) destaca que es importante lograr la autodeterminación del territorio Pasto que para ellos no es la que aparece en los documentos del Derecho Internacional Público, sino que se entiende desde la binacionalidad y desde lo Panamazónico del Nudo de Waca. De este modo se conecta lo humano y la historia de las relaciones ancestrales en la verticalidad y transversalidad (Mamian, 2000).

La cartografía panamazónica se fundamenta en el origen del territorio del Pueblo Pasto desde el mito de origen de la espiral eterna de la creación de sus cordilleras, montańas, páramos, en un constante movimiento rítmico y cíclico de dos fuerzas duales, contrarias y complementarias, que nuestros mayores conocieron como 'Perdices Poderosas' que personifican lo activo y lo pasivo, lo masculino y lo femenino. [...] De esta manera interpretamos y entendemos la Panamazonía, cuando los ríos de nuestro resguardo nos conectan con el Pacifico y con lo Amazónico, invitándonos a caminar, recorrer, hacer camino y leer el territorio en su ordenamiento y biodinámica. Si nos debemos al territorio, nuestra vida comunitaria también obedece a una planificación sujeta al ordenamiento natural y sagrado del Nudo de Waka. (Ministerio de Ambiente, 2010, p. 20)

De modo que lo Panamazónico es una concepción de las relaciones entre la Sierra, Costa y Amazonía, de sus tierras altas y bajas, que trae a la mente aquellas "relaciones internacionales" existentes entre los pueblos indígenas en el periodo precolonial. En este sentido, los Pasto no comprenden la Panamazonía tal y como es manejada por los Estados (lo que surca la Amazonia), sino como la conexión de las fronteras geoculturales de los diversos pueblos.

Entonces ahí podemos mirar a los pueblos, en este caso al pueblo Awá, que ya está al pie del monte costero al Pacifico; está el pueblo de los Pastos en los Andes, y estarían los pueblos hacia la Amazonía. Para nosotros esta es nuestra conceptualizad [sic] que queremos trabajar: estamos ubicados en la Pan Amazonía, en este caso también, hablando de la verticalidad y transversalidad, nuestras tierras son muy ricas y nuestros pueblos se han ido ubicando históricamente de acuerdo a lo que la naturaleza les ofrece. (Estacio, 2010, p. 23) 
El Plan Estratégico Binacional de los Pasto surgió como iniciativa del pueblo Pasto a través de la asociación Shaquiñan. El Plan fue presentado al gobierno de Colombia por los Pasto y este a través de diversos Ministerios y en el marco de la Comisión de Vecindad, trabajó conjuntamente con Ecuador (CONPES, 2004, p. 47).

El plan de vida de los Pastos fue reconocido en Colombia por el CONPES 3303, y además, es parte de la política pública en del país. Los temas que se vienen adelantando en el Plan Estratégico Binacional con todos los resguardos son: seguridad alimentaria, política del ordenamiento territorial, reivindicación de la cultura, de la identidad; gobierno, justicia y administración. (Castillo, 2011, p. 8)

Aunque el Plan de Vida Binacional haya tenido el apoyo de organismos externos (ONG, organismos internacionales, municipios, etc.) su construcción "se ha realizado en las comunidades mediante las "mingas de pensamiento", a partir de las cuales garantizan la concertación sobre los planes de ordenamiento territorial (Guerrero, 2008, p. 185). Del mismo modo, gracias al Convenio Interadministrativo No 054 del 2 de agosto de 2012 entre el Ministerio de Ambiente de Colombia y Shaquiñan se ratificó la propuesta de la cosmovisión Pasto, para conservar y proteger el territorio del Nudo de Waca (Ministerio de Ambiente, 2012, p. 9).

De esta manera, a partir del año 2005, se formula el Plan Binacional para el Fortalecimiento Cultural, Natural y Ambiental del Nudo de los Pastos, que establece cuatro ejes temáticos relacionados así: Territorio y Ambiente natural, Soberanía Alimentaria Medicina Natural y Desarrollo Económico, Cultura ancestral y Educación Propia, Gobierno Administración y Justicia Propia. Es así que el Gobierno Nacional, a través del Departamento Nacional de Planeación-DNP, acoge el plan binacional como una política regional desde la perspectiva como pueblo indígena, la cual se convierte en una estructura de gestión interinstitucional para el bienestar del territorio y de todos quienes lo habitamos. (Ministerio de Ambiente, 2012, p. 100)

En el marco de creación del Plan de Vida Binacional han sido importantes los encuentros con referencia a la cosmovisión y cultura. Entre los Pasto y los Inga de Colombia se celebra desde el 2002 la "Ceremonia Sagrada del Ambi Uasca", ${ }^{3}$ y fue en una de estas ceremonias donde se decidió recuperar la del Inti Raymi junto con los Pasto de Ecuador. De este modo, desde el año 2005 se están reuniendo en el Nudo de Waka para celebrar tanto el Inti Raymi (en la parte colombiana) como el Kolla Raymi en la parte ecuatoriana (Tarapues et al., 2012). Además, en estas fiestas han participado las comunidades, los cabildos, las alcaldías, la gobernación del departamento y el Ministerio de Cultura (Ministerio de Ambiente, 2010, p. 4).

La Fiesta Sagrada al Sol, permite fortalecer las comunidades sobre la conciencia de pertenecer a una cultura y a un territorio (Nudo de los Pastos). Se traduce en una manifestación ritual y de agradecimiento al Sol, la Madre Tierra y los Astros, logrando fortalecer los procesos comunitarios, agrarios, sociales y culturales, en armonía con el territorio, lo cual fortalece el arraigo de las poblaciones indígenas. Este Evento, permite no solo fortalecer el amor de las poblaciones hacia sus resguardos, sino también hacia el territorio del Nudo de Huaca (Colombia y Ecuador). [...] Todas las comunidades

\footnotetext{
${ }^{3}$ Más conocido como Yagé en la Amazonia.
} 
participan en los lugares escogidos cada año, a manera rotativa. [...] El Inti Raymi es una de las cuatro fiestas cósmicas y a través de ella se tiene la idea de fortalecer las otras tres fiestas sagradas (Kolla Raymi o fiesta femenina, fiesta de la luna, del 21 de Septiembre en el Equinoccio de Invierno, el Kapak Raymi o fiesta de los niños, del 21 de Diciembre en el Solsticio de Invierno y el Pawkar Raymi o fiesta del florecimiento, del 21 de marzo en el Equinoccio de Verano). (Tarapues S.f., pp. 8-10)

Además de los eventos del Inti Raymi y Killa Raymi, los Pasto de la Comuna La Esperanza (Tufiño) y el Resguardo Indígena de Chiles han organizado junto con la Gobernación de Nariño, el Gobierno Provincial del Carchi, la Secretaría de Agricultura de Nariño y la Alcaldía de Tulcán, entre otros, el "Ascenso Binacional Volcán Chiles" durante tres ańos consecutivos (El Norte, 2013; La Hora 2012) para impulsar la hermandad transfronteriza.

La Fundación Natura Regional (FNR) — a través del proyecto "El Medio Ambiente y la Biodiversidad como Punto de encuentro entre actores sociales de Colombia y Ecuador" - ha elaborado talleres con los Pasto para identificar los lineamientos del Plan de Ordenamiento Territorial Binacional del Nudo de los Pastos. En estos talleres se han discutido los principales problemas que existen en los ejes antes mencionados y los lineamientos y acciones propuestas para superarlos (Lucio y Castro, 2011, pp. 94-98). Algunos de los problemas que los Pasto han identificado como causantes de la fragmentación a nivel binacional son la deforestación, el mal uso de agroquímicos, la frontera que les ha hecho verse diferentes los unos a los otros y la pérdida de identidad binacional.

A su vez, en el marco del Plan Pasto Binacional se han implementado diversas iniciativas. Entre ellas se encuentra el "Proyecto de Desarrollo Territorial Transfronterizo con identidad al sur de Colombia y norte de Ecuador, Proyecto Transfronterizo Pasto" (2011-2015), en el que participan el Departamento de Nariño (municipios Cumbal, Guachucal, Santa Cruz, Aldana, Córdoba, Tuquerres y Contadero) y la Provincia de Carchi (cantones Montufar y Bolívar). Esta propuesta "organizativa y ancestral de fortalecimiento de la frontera colombo ecuatoriana" ha surgido gracias al trabajo conjunto entre el Gobierno Provincial del Carchi, la Gobernación de Nariño, Agrónomos y Veterinarios Sin Fronteras (AVSF), Shaquiñan y el CCM (Consejo de Comunas Campesinas de Montúfar), con apoyo de la Unión Europea (Castillo, 2011, p. 8).

Como resultado de esta acción se apoya el proceso de mercados locales en Cumbal (Nariño-Colombia) y la feria solidaria de productos con principios agroecológicos en San Gabriel (Carchi-Ecuador). Es importante destacar la participación de las organizaciones indígenas de Colombia y Ecuador en redes internacionales como el Movimiento Agroecológico Latinoamericano - MAELA — y la Asociación de Poblaciones de Montañas del Mundo -APMM-, dinamizando desde lo local los foros de minería y agua en Pasto y Guachaves y gracias a la articulación con organizaciones locales como la Minga Agroecológica al Sur, se pudo desarrollar una primera versión del "Diplomado en Agroecología" que contó con el apoyo de la Agencia de Desarrollo Local, y el reconocimiento a la formación de 32 Shagreros del pueblo Pasto, por parte de la Pontificia Universidad Javeriana. (Revelo, 2012, p. 8)

Una segunda iniciativa que permitió la consolidación del anterior proyecto fue la "Implementación de sistemas sostenibles de producción-conservación con principios agroecológicos, como aporte a la seguridad alimentaria de las familias y a la construcción de un desarrollo rural alternativo, que favorezca la satisfacción gradual de las necesidades humanas fundamentales de las familias 
campesinas e indígenas habitantes de la zona de frontera de Colombia y Ecuador". El proyecto se implementó en los municipios de Ricaurte, Mallama, Ipiales y Puerres del Departamento de Nariño, Colombia y en las Parroquias de Chical, Tufiño y Maldonado, del Cantón Tulcán, Provincia de Carchi, Ecuador (Revelo, 2011). En el marco de este proyecto, también se celebró la "Primera Feria Binacional saberes, sabores y semillas del campo", en la cual 35 organizaciones del espacio fronterizo compartieron "expresiones culturales, semillas y productos transformados, incluyendo el trueque, realizado por campesinos indígenas y afrodescendientes" (Revelo, 2011).

\section{Conclusiones}

Como hemos visto en el presente estudio, los Pasto han logrado fortalecer su identidad, especialmente en la parte ecuatoriana donde se encontraba más debilitada. Esto se debe a dos procesos: el primero a nivel estatal (su ingreso a ECUARUNARI) y el segundo, a nivel transfronterizo, a partir del proceso binacional formulado en las mingas de pensamiento del pueblo Pasto.

Al tomar como base la "Nación Pasto" y su identidad como pueblo, trascienden la frontera reivindicando el territorio con los conceptos del Nudo de Waka y la Panamazonía. Aunque hayan sido apoyados a través de redes articuladas con ONG, municipios u organismos internacionales todavía son vistos como sujetos folklóricos más que como sujetos políticos. De todas maneras, cuentan con el documento CONPES 33 de Colombia que validó el Plan Binacional Pasto y el Contrato Interadministrativo no 054 entre Shaquiñan y el Ministerio de Ambiente y Desarrollo, que valida la cosmovisión Pasto en los proyectos de integración de frontera como políticas públicas.

Asimismo, estos son factores que permiten considerar a los indígenas Pasto como sujetos activos en la construcción de la frontera. También podemos afirmar que, en cierta medida, disponen de capacidad de diálogo con otros sectores, y por lo tanto, mayor capacidad de financiación para realizar iniciativas transfronterizas. Asimismo, el puente Internacional de Rumichaca, lugar por donde se mueve el comercio, explica en cierta medida el interés del desarrollo de esta zona. Sin embargo, los Pasto han manifestado que esta área sigue estando abandonada y que se ha impuesto la fragmentación del territorio a través de las lógicas institucionales y territoriales, las divisiones político-administrativas, la extracción de recursos naturales y el proyecto IIRSA.

A través de su Plan de Vida Binacional, han identificado los siguientes ejes sobre los que es necesario trabajar: el Territorio ancestral y ambiente natural, la cultura ancestral y la educación propia, la soberanía alimentaria, medicina natural y desarrollo económico, y; el gobierno, administración y justicia. Hasta la fecha, a través de los proyectos implementados en el lugar, se está apoyando la reconstrucción de sus territorios, el intercambio de semillas y las ferias en la frontera; aunque sobre todo se reivindica el tema de la identidad y la cosmovisión a través de la celebración del Inti Raymi y Kolla Raymi, que impulsan el fortalecimiento de las comunidades Pasto así como sus aspiraciones políticas transfronterizas.

Por último, a través de los conceptos de la Panamazonía y del Nudo de Waka los Pasto desarrollan una visión alternativa del proceso de integración fronteriza desde la integralidad del territorio. Asimismo, la noción de Panamazonía permite vincular al resto de los pueblos indígenas que se encuentran en los espacios fronterizos de la Sierra, Costa y Amazonia y superar el modelo andinocéntrico existente en Ecuador y Colombia. 


\section{Referencias}

1. Andrade, P. (2002). Diagnóstico de la frontera EcuadorColombia. Comentario Internacional: Revista del Centro Andino de Estudios Internacionales, 4, 189-240.

2. Arturo, M. (2013). Promueven la Comunidad Binacional Transfronteriza. HSBNoticias. Recuperado de <http://hsbnoticias.com/vernoticia.asp?wplaca=63645>

3. Calero, L. (1991). Pastos, Quillacingas y Abades, 15351700. Bogotá D.C.: Fondo de Promoción de la Cultura del Banco Popular.

4. Calpa, L. (2011) El pueblo indígena de los Pastos. En O. Garzon, Migración Forzada en la zona sur-occidente: el caso del pueblo indigena de 'los Pastos'. X Congreso Nacional de Sociología, Cali - Colombia, noviembre 2, 3 y 4 de 2011.

5. Campion, M. (2014). La organización indígena transfronteriza en los márgenes del Ecuador. Perspectivas desde la Sierra, Costa y Amazonía. Tesis Doctoral. Manuscrito sin publicar. En prensa.

6. Carrión, F. (2010). En el límite de la vida: La violencia fronteriza. Boletin Fronteras, 3, 6-9.

7. Carrión, F. (2011). Dilemas de la seguridad ciudadana en las zonas de frontera de Ecuador. En J. Espín (Ed.). Relaciones fronterizas: encuentros y conflictos (pp. 51-61). Quito: FLACSO-Sede Ecuador/IDRC-CRDI.

8. Castillo, T. (2011). Fortaleciendo la unidad del pueblo de los Pastos. Voces de Nariño, 14(8).

9. Castro, J. (2011). Elementos de reflexión para una agenda ambiental en la frontera entre Colombia y Ecuador. Rostros y Rastros, 7, 35-44.

10. CONAIE (1989). Las nacionalidades indigenas en el Ecuador: Nuestro proceso organizativo. Quito: Ediciones Abya-Yala.

11. CONPES (2004). Estrategias para apoyar el desarrollo integral del Departamento de Nariño. Dirección Nacional de Planeación. Recuperado de <https://www.dnp.gov.co/ Portals/0/archivos/documentos/.../3303.pdf>

12. Delgado, J. (2004). La crónica de los Pastos. Quito: Ediciones Abya Yala.

13. El Norte (2013). 'Cumbres por la paz' en la frontera. $E l$ Norte. Recuperado de <http://www.elnorte.ec/carchi/actualidad/38996-\%E2\%80\%98cumbres-por-la-paz\%E2\% 80\%99-en-la-frontera.html>

14. Espinosa, R. (2008). Respuestas de Roque Espinosa. En S. Ramírez (Ed.). Ecuador: miradas binacionales (pp. 391394). Bogotá D.C.: Academia Diplomática de San CarlosMinisterio de Relaciones Exteriores de Colombia-Instituto de Estudios Políticos y Relaciones Internacionales.

15. Espinosa, R. (2009) El enclave cocalero colombo-ecuatoriano. En G. Jaramillo (Ed.). Construyendo Puentes entre Ecuador y Colombia (pp. 81-130). Quito: FLACSO/OEA/PNUD.

16. Estacio, R. (2010). Los Pueblos indígenas en la frontera colombo-ecuatoriana. En Fundación Natura Regional, Taller binacional: Colombia y Ecuador: vecinos y socios en el desarroIlo sostenible. Quito 22-23 de julio de 2010

17. Gobernadores del Pueblo Pasto (2011). Mandato frente a las acciones de exploración, extracción y explotación minera en territorio indígena. Observatorio de Conflictos Mineros de América Latina. Recuperado de <http://www.conflictosmineros.net/contenidos/10-colombia/9548-mandato-frente-a-las-acciones-de-exploracion-extraccion-y-explotacion-minera-en-territorio-indigena>

18. Grimson, A. (2000). Pensar fronteras desde la frontera. Nueva Sociedad, 170, 164-166.

19. Groot de Mahecha, A. y Hooykaas, E. (1991). Intento de delimitación del territorio de los grupos étnicos Pastos y Quillacingas en el altiplano nariñense. Bogotá D.C.: Icono Editores.

20. Guerrero, J. (2008). Situación territorial del pueblo de los Pasto de Nariño. En J. Houghton (Ed.). La Tierra contra la muerte. Conflictos territoriales de los pueblos indigenas en Colombia, (pp. 199-222). Bogotá D.C.: CECOIN.

21. Hocquengheim, A. y Durt, E. (2006) La frontière PérouEquateur: enjeu mondial, empreintes locales. En J. Lombard, E. Mesclier y S. Velut (Eds.). La mondialisation côté Sud: acteurs et territoires (pp. 307-320). Paris: IRD Editions.

22. La Hora. (2011). Primera comunidad Pasto nace en Carchi. La Hora. Recuperado de <http://www.lahora.com. ec/index.php/noticias/show/1101207939/-1/Primera_comunidad_Pasto_nace_en_Carchi.html\#.UvjMxpyWkrs>

23. La Hora (2012). Encuentro binacional de turismo por la paz. La Hora. Recuperado de <http://www.lahora.com.ecl index.php/noticias/show/1101355078/-1/Encuentro_binacional_de_turismo_por_la_paz_html\#.U2iOs5wSOhS>

24. Lucio, G. y Castro, J. (2011). Agenda positiva binacional colombo-ecuatoriana sobre medioambiente, biodiversidad y desarrollo sostenible. Quito: CAF/Fundación Natura.

25. Mamián, D. (2000). Los Pastos. En VV.AA Geografía Humana de Colombia. Región Andina Central. Tomo IV. Volumen I. Bogotá D.C.: Instituto Colombiano de Cultura Hispánica.

26. Mamián, D. e Inuca, B. (2011). Construyendo la frontera fuera del tiempo en que vivimos. La Minga de Pensamiento del pueblo Pasto. Quito: Fundación Natura Regional/CAF.

Bogotá, D. C. - Colombia - Volumen 13, Número 16 (julio-diciembre) Año 2015 
27. Ministerio de Ambiente y Desarrollo Sostenible y Shaquiñan (2012). Convenio no 054. Agenda ambiental en el territorio del Nudo de la Wuaka o de los Pastos para la permanencia de la vida y la cultura. Recuperado de <http:// www.minambiente.gov.co/documentos/documentosOrdenamiento/agenda_ambiental/051113_agenda_amb_ nudo_wuaka.pdf>

28. Montenegro, R. (2008a). Decisiones con las fronteras, no a pesar de ellas. En S. Ramírez (Ed.). Ecuador: miradas binacionales (pp. 375-387). Bogotá D.C.: Academia Diplomática de San Carlos-Ministerio de Relaciones Exteriores de ColombiaInstituto de Estudios Políticos y Relaciones Internacionales.

29. Montenegro, R. (2008b). Respuestas de Ricardo Montenegro. En S. Ramírez (Ed.). Ecuador: miradas binacionales (pp. 395397). Bogotá D.C.: Academia Diplomática de San CarlosMinisterio de Relaciones Exteriores de Colombia-Instituto de Estudios Políticos y Relaciones Internacionales.

30. Navarro, A. (2009). Desarrollo binacional visto desde Nariño. En G. Jaramillo (Ed.). Construyendo Puentes entre Ecuador y Colombia (pp. 175-181). Quito: FLACSO/ OEA/PNUD.

31. Obando, A. (2009). Plan frontera sur: construir afinidades y bienes comunes. En S. Ramírez (Ed.). Colombia Ecuador. Construyendo puentes. Politica de paz y desarrollo para la frontera (pp. 66-74). Bogotá D.C.: Universidad Nacional de Colombia.

32. Pineda, J. y Krainer, A. (2012). Los territorios indígenas de la Costa y Amazonía ecuatoriana: las historias no narradas de la nación. En J. Pineda y A. Krainer (Coords.). Periferias de la periferia: procesos territoriales indigenas en la Costa y la Amazonia ecuatoriana (pp. 11-22). Quito: FLACSO.

33. Ramírez, S. (2008a) Realidades económicas fronterizas y binacionales. En S. Ramírez (Ed.). Una mirada al Ecuador (pp. 209-211). Bogotá D.C.: Universidad Nacional de Colombia. Instituto de Estudios Políticos y Relaciones Internacionales. Ministerio de Relaciones Exteriores de Colombia.

34. Ramírez, S. (2008b). Dinámicas transfronterizas y encrucijadas para los Estados. En S. Ramírez (Ed.). Una mirada al Ecuador (pp. 359-362.). Bogotá D.C.: Universidad Nacional de Colombia. Instituto de Estudios Políticos y Relaciones Internacionales. Ministerio de Relaciones Exteriores de Colombia.

35. Ramírez, S. (2010). Elementos dinamizadores de las relaciones colombo ecuatoriana. En Fundación Natura Regional. Taller binacional: Colombia y Ecuador: vecinos y socios en el desarrollo sostenible. Quito 22 y 23 de julio de 2010.

36. Ramos, I. (2008). Carchi, las fronteras desconocidas. En R. Espinosa (Ed.) Las fronteras con Colombia (pp. 45228). Quito: Corporación Editora Nacional y Universidad Andina Simón Bolívar.

37. Revelo, J. (2011). Implementación de sistemas sostenibles de producción- de la zona de frontera de Colombia y Ecuador. CAN. Recuperado de <http://www.comunidadandina.org/rural/proyectos/ADC_resultados.pdf> [25.11.2014]

38. Revelo, V. (2012). Un aporte para el fortalecimiento de la integración del Pueblo Pasto en la zona de frontera Colombo Ecuatoriana. Voces de Nariño, 18(8).

39. Rivera, G. (2010). Propuestas concretas para fortalecer la buena vecindad colombo-ecuatoriana. Aldea Mundo, 15(29), 79-82.

40. Romero, J. (2010). Iniciativas binacionales de la sociedad civil colombo-ecuatoriana en la costa del Pacífico y los Andes. Taller Binacional. Colombia y Ecuador: vecinos y socios en el desarrollo sostenible, 22 y 23 julio de 2010. Quito: Fundación Regional Natura.

41. Santacruz, L. (2009). Interculturalidad, educación y frontera. Repensando maneras otras desde las fronteras de Ecuador y Colombia. Decisio, 22, 21-26.

42. Tarapues, et al. (2012). El Nudo de Waka o de los Pastos. Departamento Nacional de Planeación, Gobierno de Colombia. Recuperado de <https://www.dnp.gov.co/LinkClick.aspx:fileticket=5y29uhOAQFI\%3D\&tabid=379>

43. Tarapues, E. (2006). Un espacio binacional para los pueblos de montańa de los pastos. VV.AA. Nous, peuples de montagne. Somos pueblos de montaña. We, the mountain people (pp. 132-135). Paris: APMM.

44. Tarapues, E. (2009). Plan binacional Pueblo Pastos. En S. Ramírez (Coord.). Colombia-Ecuador construyendo puentes. Politicas de paz y desarrollo para la frontera (pp. 81-84). Bogotá D.C.: Universidad Nacional de Colombia/OEA/ PNUD.

45. Tarapues, E. (2013) El Plan binacional del Pueblo de los Pasto, [Entrevistado por M. Campion]. Pasto, 27 de noviembre de 2013.

46. Tarapues, E. (S.f.) Fiesta Sagrada al Sol Inti Raymi. Convocatoria al Seminario sobre desarrollo local y OVOP. Recuperado de <https://ovop.dnp.gov.co/Portals/1/Fichas\%20por\%20iniciativa/Pueblo\%20de\%20los\%20Pastos/1_Ficha_Ensayo_ Inti\%20Raymi.pdfs 
47. Tarapues, E. y Rico, A. (2012). Gobernabilidad y aspectos culturales en resolución de conflictos ambientales. En B. Londońo, L. Güiza y L. Muñoz (Eds.). Conflictos ambientales en Colombia. Retos y perspectivas desde el enfoque de DDHH y la participación ciudadana (pp. 67-72). Bogotá D.C.: Editorial Universidad del Rosario.

48. Valenzuela, L. (2007). Ámbitos de integración fronteriza colombo-ecuatoriana. En S. Ramírez y C. Montúfar (Eds.). Colombia-Ecuador. Cercanos y distantes (pp. 209-
210). Bogotá D.C.: Universidad Nacional de Colombia/Universidad Andina Simón Bolívar.

49. Velasco, P. (2009). La integración fronteriza desde lo local. En G. Jaramillo (Ed.). Construyendo Puentes entre Ecuador y Colombia (pp. 181-196). Quito: FLACSO/OEA/PNUD.

50. Vieira, E. (2008). La formación de espacios regionales en la integración de América Latina. Bogotá D.C.: Convenio Andrés Bello. 